\title{
AVALIAÇÃO DOS PARÂMETROS SISTÊMICOS E BIOQUÍMICOS EM HOMENS TRANSGÊNEROS PÓS TERAPIA HORMONAL
}

\section{ARTIGO DE REVISÃo}

MARTINS, Maria Isabel Morgan ${ }^{1}$

OLIVEIRA, Jason Sant'Ana de ${ }^{2}$

SANTOS, Ana Maria Pujol Vieira dos ${ }^{3}$

MARTINS, Maria Isabel Morgan. OLIVEIRA, Jason Sant'Ana de. SANTOS, Ana Maria Pujol Vieira dos. Avaliação dos parâmetros sistêmicos e bioquímicos em homens transgêneros pós terapia hormonal. Revista Científica Multidisciplinar Núcleo do Conhecimento. Ano 05, Ed. 11, Vol. 19, pp. 99-114. Novembro de 2020. ISSN: 24480959, Link de acesso:https://www.nucleodoconhecimento.com.br/saude/avaliacaodos-parametros

\section{RESUMO}

A transexualidade refere-se ao indivíduo cujo gênero não está alinhado com seu sexo biológico, essa é uma condição que desencadeia um sentimento de não pertencimento, e vem aumentando sua incidência nos últimos anos. Tem origem desconhecida, e estudos atuais apontam que vários fatores biológicos podem estar ligados à ocorrência da mesma. Indivíduos trans masculinos são aqueles que

${ }^{1}$ Doutorado em Ciências Biológicas (Fisiologia). Mestrado em Ciências Biológicas (Fisiologia). Graduação em Ciências Habilitação Em Biologia.

${ }^{2}$ Acadêmico do Curso de Biomediciana - Universidade Luterana do Brasil/ULBRA Canoas - RS.

${ }^{3}$ Doutorado em Fitotecnia. Mestrado em Microbiologia Agrícola e do Ambiente. Especialização em Educação a Distância. Aperfeiçoamento em Aperfeiçoamento. Graduação em Ciências Biológicas. 
nasceram com o sexo biológico feminino, porém não se identificam com esta designação. A terapia de primeira escolha é a administração de testosterona exógena aplicada via intramuscular. O objetivo do presente estudo foi descrever os parâmetros sistêmicos e bioquímicos de homens trans pós terapia hormonal, a partir da análise de artigos que destacam as alterações sistêmicas desencadeadas pela administração da testosterona. A inversão dos valores de referência do feminino para o masculino, em diversos parâmetros sistêmicos, pôde ser observada após a realização do cálculo das médias dos resultados reportados pela literatura, apontando os mecanismos pelos quais a testosterona age no organismo.

Palavras-Chave: 17-Hidroxiesteroide Desidrogenases, transexualidade, hormônios, terapia de reposição hormonal.

\section{INTRODUÇÃO}

\subsection{SOBRE A DISFORIA DE GÊNERO}

A transexualidade, também chamada de transtorno de identidade de gênero (TIG) ou ainda disforia de gênero (DG), está classificada no CID F64-0 ("CID10 Código Internacional de Doenças", 2020) e refere-se ao indivíduo cujo sexo biológico não se alinha com sua identidade de gênero, ou seja, ele presencia um gênero diferente do que the foi designado ao nascer (BURKE et al., 2017; SARASWAT; WEINANDSAFER, 2015; UNGER, 2016). No caso dos homens trans, são indivíduos que nasceram biologicamente do sexo feminino, mas se identificam com o gênero masculino.

Sexo biológico, gênero e orientação sexual são coisas diferentes. Identidade de gênero é sobre auto identificação, como individualmente a pessoa experiência sua existência. Orientação sexual se trata da atração perante os outros gêneros, indivíduos transgêneros podem ser heterossexuais, bissexuais ou homossexuais, sem que isto afete sua identidade de gênero e, por fim, sexo biológico está ligado ao fator genital e cromossômico, sendo o que define toda a questão fisiológica envolvida (GUPTA; IMBOREK; KRASOWSKI, 2016; ADRIAANSEN et al., 2017). 


\subsection{ETIOLOGIA}

\subsubsection{FATORES NEUROLÓGICOS}

Em um estudo que analisava alterações neurológicas nesta população, foi descoberto que alguns homens transgêneros analisados apresentavam a estrutura cerebral compatível com a estrutura de homens cis (que se identificam com seu sexo designado ao nascer), enquanto outros apresentavam sua estrutura cerebral diferente de ambos os sexos dos grupos controle (BURKE et al., 2017). Outros estudos, como o de Uribe et al. (2020), em indivíduos com DG reportaram que foram encontradas alterações neurológicas em locais do cérebro responsáveis pela percepção corporal, em comparação com o grupo controle, composto por homens cisgêneros (URIBE et al., 2020).

Swaab et al. (2011) propôs que a incompatibilidade entre gênero e sexo biológico poderia se dar pelo fato de que a diferenciação sexual do cérebro ocorre antes da diferenciação das gônadas sexuais no começo da vida fetal (BAO; SWAAB, 2011). Ainda na esfera neurológica, Yokota et al. (2005) reportou que o padrão do corpo caloso em pessoas com DG é mais próximo a pessoas do mesmo gênero do que comparado ao de pessoas do mesmo sexo biológico (YOKOTA; KAWAMURA; KAMEYA, 2005).

\subsubsection{FATORES GENÉTICOS}

Quanto aos fatores genéticos que estão sendo estudados para entender o desenvolvimento da condição, o mais apontado está ligado ao gene CYP17 (SARASWAT; WEINANDSAFER, 2015; BENTZ et al., 2008). O gene CYP17 é responsável pela codificação da síntese de uma proteína da família do citocromo P450, a CYP17A1, que está ligada à síntese de precursores de hormônios esteroides, como a testosterona e o estrogênio, que são necessários para o normal desenvolvimento e reprodução dos seres humanos (BENTZ et al., 2008). 
Um estudo de caso controle que contou com a participação de cento e cinquenta e um participantes com DG apontou uma mutação no gene CYP17 como uma teórica causa da condição. O CYP17 A2 T>C é um polimorfismo de nucleotídeo único funcional, que está associado com a elevação no soro e plasma dos níveis de estradiol, progesterona e testosterona (BENTZ et al., 2008). Os resultados da pesquisa apontaram que dos 151 partícipes (49 deles sendo trans homens), o alelo mutante encontrava-se estatisticamente em maior associação com os indivíduos trans masculinos e não com participantes trans mulheres (biologicamente designadas ao sexo masculino ao nascer). A distribuição alélica nas mulheres trans foi equivalente aos controles masculinos cis gêneros, enquanto essa distribuição na parcela de homens trans não seguiu um padrão de gênero específico, tendo sua distribuição equivalente também ao grupo dos homens cis (SARASWAT; WEINANDSAFER, 2015; BENTZ et al., 2008).

\subsubsection{FATORES DE EXPOSIÇÃO PRÉ-NATAL}

Fatores de exposição pré-natal também foram avaliados, foram realizados estudos em uma amostra de quinhentos participantes com DG, todos eles expostos durante a vida fetal a um medicamento estrógeno para prevenção de aborto, Diethylstilbestrol (DES), destes 500 partícipes, 150 apresentavam disforia de gênero severa (SARASWAT; WEINANDSAFER, 2015).

\subsection{EPIDEMIOLOGIA}

O número de pacientes com DG que procuram auxílio e tratamento vêm aumentando com o passar das décadas (UNGER, 2016; GUPTA; IMBOREK; KRASOWSKI, 2016) e, segundo um levantamento de dados, que foi considerado o maior estudo recente sobre a prevalência da transexualidade, foi reportado que a prevalência é de aproximadamente 1 homem trans para 40.000 pessoas cis, e de 1 mulher trans para cada 15.000 pessoas cis (UNGER, 2016).

Porém, como muitos estudos de prevalência necessitam de uma base de dados clínica, e mesmo com o aumento no número de indivíduos com DG uma grande 
quantidade dos mesmos optam por realizar a terapia hormonal em casa, sem comparecer a órgãos de saúde, a contabilização do número de casos é complicada, deixando assim transparecer apenas uma pequena parcela da verdadeira prevalência (UNGER, 2016).

O objetivo do presente estudo, foi descrever os parâmetros sistêmicos e bioquímicos de homens trans pós terapia hormonal. A partir de uma revisão da literatura foram investigados os parâmetros: Série vermelha ( $\mathrm{Hb}, \mathrm{HTC}$, contagem de eritrócitos), contagem de leucócitos, creatinina, enzimas hepáticas (ALT, ALT, GGT), perfil lipídico (colesterol total, HDL, LDL, triglicerídeos) dosagem de glicose em jejum e perfil hormonal (testosterona total e estradiol). Com base nestes dados analisados foi realizado o cálculo da média dos valores obtidos, para fins de análise dos perfis deste grupo.

\section{MATERIAIS E MÉTODOS}

\subsection{DELINEAMENTO DO ESTUDO}

O presente trabalho se trata de uma Revisão de Literatura baseada na pesquisa e análise de obras bibliográficas e de artigos científicos referentes ao assunto delimitado, disponíveis e de livre acesso nos bancos de dados científicos dos indexadores PubMed e Scientific Electronic Library Online (Scielo). No intuito de promover uma revisão ampla do assunto, foram selecionadas publicações nacionais e internacionais preferencialmente dos últimos dez anos, que abordem as alterações sistêmicas ocorrentes em indivíduos trans masculinos pós terapia hormonal.

Os dados analisados foram coletados de um grupo de artigos, 16 deles tendo sido publicados dentro do período de 2015-2020, usando como palavras-chave os seguintes termos em inglês: 17-Hydroxysteroid Dehydrogenases, Transsexualism, Hormones e Hormone Replacement Therapy. Após a coleta dos resultados relatados pela literatura para os parâmetros bioquímicos e sistêmicos, foi realizado um cálculo da média desses dados para fins de análise das alterações pós terapia hormonal. 


\section{RESULTADOS}

\subsection{TERAPIA}

\subsubsection{ERROS ANALÍTICOS}

É possível perceber o aumento do número de pacientes com DG nas clínicas e laboratórios, pois cada vez mais esta população procura por auxílio médico para realizar a terapia hormonal (UNGER, 2016). Em sua grande maioria, os sistemas dos laboratórios de análises clínicas contemplam apenas o sexo biológico do paciente em seus formulários, este sistema binário não permite a inserção de dados de gênero. Isto é um problema, pois se o usuário já está em terapia hormonal, os valores de referência já não serão os mesmos, e os aparelhos de análise automatizados configurados com os valores de referência masculinos e femininos, liberarão as amostras destes pacientes como se apresentassem resultados anormais em certos marcadores (UNGER, 2016; ADRIAANSEN et al., 2017).

Nesta situação, os marcadores mais afetados são aqueles que se utilizam da informação do sexo para liberarem os resultados, como por exemplo testes de enzimas hepáticas, creatinina e hematócrito (GUPTA; IMBOREK; KRASOWSKI, 2016), deixando com o analista a responsabilidade de reconhecer se este resultado realmente está alterado ou não (GUPTA; IMBOREK; KRASOWSKI, 2016; ADRIAANSEN et al., 2017).

No Brasil, nos serviços do SUS e garantido por lei, a população trans tem direito à utilização de nome social, nome escolhido pelo indivíduo. A Portaria no 1.820/2009 diz que o usuário deve ser identificado por prenome e sobrenome civil, porém, em todos os documentos deve existir um campo para o uso de nome social (SILVA et al., 2017). Este direito facilita a vida do usuário transgênero, pois pode também ser utilizado em laboratórios, permitindo que o analista tenha uma visão mais ampla da situação deste usuário no momento de verificar os flags das máquinas (SILVA et al., 2017). 


\subsubsection{TERAPIA HORMONAL}

Os exames clínicos fazem parte da fase pré tratamento e também são utilizados para acompanhar a saúde do paciente (UNGER, 2016). A terapia utilizada no tratamento de homens com DG é a administração de testosterona exógena, sendo mais utilizada neste tratamento em sua forma para aplicação intramuscular, no entanto, a medicação também pode ser administrada via oral, subcutânea e transdermal (UNGER, 2016; ADRIAANSEN et al., 2017; GOOREN, 2014). O princípio do tratamento com testosterona $(\mathrm{T})$ é o mesmo do tratamento dado à homens que realizam reposição hormonal (UNGER, 2016).

O caminho para se dar início ao tratamento varia ao redor do mundo, mas geralmente começa com o encaminhamento de um profissional da saúde mental para um endocrinologista, sendo ele o responsável por prescrever a medicação (UNGER, 2016; GOOREN, 2014). Os objetivos da terapia hormonal (TH) com T são suprimir as características sexuais femininas secundárias, e desenvolver características masculinas e virilização ao usuário.

O intervalo de administração da $T$ varia de paciente para paciente, e leva em consideração o objetivo do tratamento, tempo de tratamento e fatores clínicos individuais, sendo este intervalo inicialmente a cada quatorze dias, e após algum tempo, vinte e um dias (UNGER, 2016; GOOREN, 2014; CHIPKIN;KIM, 2017). A T age no organismo de maneira a estimular uma segunda puberdade, e toda essa transição pode demorar até cinco anos para ser completa, porém as mudanças são dependentes do hormônio, podendo algumas delas retrocederem na ausência do mesmo. Logo, a terapia deve ser realizada ao longo da vida (GORTON; ERICKSONSCHROTH, 2017).

\subsection{ALTERAÇÕES SISTÊMICAS}

\subsubsection{ALTERAÇÕES MORFOFISIOLÓGICAS}


Os receptores androgênicos estão amplamente distribuídos por todo o corpo e, por conta disto, a terapia com testosterona tem diversos efeitos em vários sistemas do corpo, afetando aspectos físicos, fisiológicos e psicológicos de quem se submete a este tratamento (IRWIG, 2017).

A literatura relata que as mudanças físicas mais comuns estão ligadas à essa segunda puberdade. No início da TH (1-3 meses) as primeiras mudanças percebidas são o aumento da oleosidade da pele, aumento da massa muscular na região superior, gerando também um aumento de força física, neste período também ocorre a redistribuição da gordura corporal para um padrão masculino, a interrupção do ciclo menstrual pode ocorrer nestes primeiros três meses (SILVA et al., 2017).

A partir dos três meses, as mudanças físicas ficam mais evidentes, sendo este período marcado pelo aparecimento de pelos faciais e corporais e alteração da voz, que irá começar a falhar e se apresentará rouca até ocorrer a definição das cordas vocais para um tom mais grave e másculo (ADRIAANSEN et al., 2017; SILVA et al., 2017; CHIPKIN;KIM, 2017; GORTON; ERICKSON-SCHROTH, 2017).

A partir do primeiro ano de TH ocorre o espessamento dos pelos faciais, a alopecia androgenética também é muito relatada neste período, o cabelo começa a cair e cresce novamente em um padrão masculino por conta da interação da $T$ com as glândulas pilossebáceas (ADRIAANSEN et al., 2017). Passada esta fase inicial da TH, iniciam-se as mudanças mais tardias, que são a atrofia do epitélio vaginal, aumento do clitóris (em média 5 centímetros) e o assentamento definitivo das cordas vocais (ADRIAANSEN et al., 2017; GORTON; ERICKSON-SCHROTH, 2017).

Indivíduos que iniciam o tratamento com idade mais avançada já apresentam um grau maior de feminilização definitiva que não podem ser revertidas com a $T$, como resultado, eles poderão ser mais baixos (diferença média de altura de até 12 centímetros), apresentar o padrão de distribuição de gordura mais feminino e também podem ter o quadril mais largo que o de um homem cis (ADRIAANSEN et al., 2017; GOOREN, 2014). 


\subsubsection{ALTERAÇÕES HEMATOLÓGICAS}

A eritropoiese é um dos muitos mecanismos do corpo que sofrem alterações por conta da administração exógena da testosterona. Estudos sugerem que os androgênicos agem indiretamente como estimulantes da produção de eritropoietina e diretamente na eritropoiese na medula óssea. Níveis maiores de testosterona no plasma sanguíneo são constantemente associados à valores maiores de hematócrito e hemoglobina, estes valores, porém, permanecem dentro dos valores de referência masculinos (homens cis) esperados (IRWIG, 2017; VELHO et al., 2017).

Realizando o cálculo da média dos dados dos artigos analisados que relatavam alterações na hemoglobina $(\mathrm{Hb})$ e hematócrito $(\mathrm{HTC})$, obtiveram-se os resultados apresentados na Tabela 1.

Levando-se em consideração os valores de referência para estes parâmetros como sendo a média da população brasileira adulta ("MSD Manuals", 2020), o resultado de 14,09 $\mathrm{g} / \mathrm{dL}$ para $\mathrm{Hb}$ encontrado estaria dentro do esperado em ambos os valores de referência, não havendo risco à saúde caso a interpretação do teste fosse realizada conforme o valor de referência feminino ou masculino, assim como o resultado do hematócrito, que obteve uma média compatível com ambos os valores de referência.

Quanto aos valores relacionados à contagem celular de eritrócitos e leucócitos, os valores basais e pós terapia hormonal de um grupo estudado também podem ser vistos na Tabela 1 (VITA et al., 2018).

Em ambas as contagens foi possível visualizar um aumento nas séries branca e vermelha (VITA et al., 2018), condizentes com o quadro de estimulação da produção de eritropoietina e da ação dos hormônios na medula óssea (IRWIG, 2017).

Tabela 1: Valores de hemoglobina, hematócrito e contagem de leucócitos e eritrócitos pré e pós terapia hormonal com testosterona. 
CONHECIMENTO https://www.nucleodoconhecimento.com.br

\begin{tabular}{|c|c|c|c|c|}
\hline $\begin{array}{l}\text { Perfil } \\
\text { Sistêmico }\end{array}$ & & $\begin{array}{l}\text { Valores de } \\
\text { Referência }\end{array}$ & $\begin{array}{l}\text { Valores } \\
\text { Pré TH }\end{array}$ & $\begin{array}{l}\text { Valores Pós } \\
\mathrm{TH}\end{array}$ \\
\hline Hemoglobina & $\begin{array}{l}\text { (Jacobeit et al, } \\
2007 \text { ) } \\
\text { (Mueller et al, } \\
2010 \text { ) } \\
\text { (Jacobeit et al, } \\
2009 \text { ) } \\
\text { (Mueller et al, } \\
2010 \text { ) } \\
\text { (Pelusi et al, } \\
2014 \text { ) } \\
\text { (Quirós et al, } \\
2015 \text { ) Média }\end{array}$ & $\begin{array}{l}\text { Masculino: } \\
\text { 17g/dL } \\
\text { Feminino: } \\
\text { g/dL }\end{array}$ & & $\begin{array}{ll}14,45 & \mathrm{~g} / \mathrm{dL} \\
14,0 & \mathrm{~g} / \mathrm{dL} \\
14,8 & \mathrm{~g} / \mathrm{dL} \\
13,9 & \mathrm{~g} / \mathrm{dL} \\
13,95 & \mathrm{~g} / \mathrm{dL} \\
13,8 & \mathrm{~g} / \mathrm{dL} \\
13,45 & \mathrm{~g} / \mathrm{dL} \\
14,4 & \mathrm{~g} / \mathrm{dL} \\
\mathbf{1 4 , 0 9} \mathbf{g} / \mathrm{dL}\end{array}$ \\
\hline Hematócrito & $\begin{array}{l}\text { (Jacobeit et al, } \\
2007 \text { ) } \\
\text { (Mueller et al, } \\
2010 \text { ) } \\
\text { (Jacobeit et al, } \\
2009 \text { ) } \\
\text { (Mueller et al, } \\
2010) \\
\text { (Pelusi et al, } \\
2014 \text { )(Quirós et } \\
\text { al, } \\
\text { Média }\end{array}$ & $\begin{array}{l}\text { Masculino: } 41-51 \\
\text { Feminino: } 36-47 \%\end{array}$ & & $\begin{array}{l}42,65 \% \\
43,87 \% \\
43,5 \% \\
42,95 \% \\
41,25 \% \\
41,60 \% \\
40,75 \% \\
\text { Não Avaliado } \\
42,36 \%\end{array}$ \\
\hline Leucócitos & Vita et al, 2018 & $4,5-11 \times 10^{3}$ & $7.4 \pm 1$ & $7.4 \pm 1.6$ \\
\hline Eritrócitos & Vita et al, 2018 & $4,2-5,9 \times 10^{6}$ & $4.4 \pm 0.4$ & $4.8 \pm 0.4$ \\
\hline
\end{tabular}

Fonte: elaborada pelo próprio autor.

\subsubsection{ALTERAÇÕES BIOQUÍMICAS}




\section{Creatinina}

Pacientes transmasculinos têm tendência a apresentarem valores de creatinina maiores após a terapia hormonal, já que com a administração de testosterona exógena ocorre um aumento considerável da massa muscular, que está diretamente relacionada aos níveis de creatinina (VITA et al., 2018; GANDHI; MEDEIROS; SHAH, 2020). Os valores médios encontrados em estudos recentes sobre este parâmetro em homens transgêneros ficaram entre $0.73 \pm 0.03$ em seu nível basal Pré T e $0.82 \pm 0.04$ após 18 meses em terapia hormonal (FERNANDEZ; TANNOCK, 2016). Os valores de referência para este parâmetro são de $0,7-1,3 \mathrm{mg} / \mathrm{dL}$ no soro.

\section{Enzimas Hepáticas}

As enzimas hepáticas são outro parâmetro que apresentam alterações significativas com a $\mathrm{TH}$, seu aumento é condizente com a inversão dos valores de referência femininos para os masculinos, porém sem superar tais valores de referência, ficando fora do patamar patológico. Os riscos da TH sobre a função hepática estão mais relacionados com a administração do hormônio via oral do que com a administração via intramuscular, por este motivo, esta é a via de administração de primeira escolha do fármaco (FERNANDEZ; TANNOCK, 2016). Os valores médios encontrados para este parâmetro podem ser vistos na Tabela 2.

\section{Perfil Lipídico}

Muitos estudos que analisaram amostras de pacientes trans masculinos relataram a diminuição do HDL e o aumento do LDL, assim como um aumento desfavorável do triglicerídeo após um ano de administração intramuscular de testosterona (IRWIG, 2017; FERNANDEZ; TANNOCK, 2016). Os valores médios encontrados podem ser visualizados na Tabela 2. Mesmo com o dito aumento de triglicerídeos e colesterol LDL, os resultados ainda estão dentro dos valores de referência para estes parâmetros.

Tabela 2: Valores de enzimas hepáticas pré e pós terapia hormonal, dosagem do perfil lipídico pós terapia hormonal. 


\begin{tabular}{|c|c|c|c|c|}
\hline Perfil Sistêmico & & $\begin{array}{l}\text { Valores de } \\
\text { Referência }\end{array}$ & $\begin{array}{l}\text { Valores Pré } \\
\text { TH }\end{array}$ & $\begin{array}{l}\text { Valores Pós } \\
\text { TH }\end{array}$ \\
\hline $\begin{array}{lr}\text { Alanina } & \text { amino } \\
\text { transferase } & (\text { ALT })\end{array}$ & $\begin{array}{l}\text { (Velho et al, } \\
\text { 2017). }\end{array}$ & 0-35 U/L & $14,4 \pm 4,2$ & $18,3 \pm 8,2$ \\
\hline $\begin{array}{ll}\text { Aspartato } & \text { amino } \\
\text { transferase } & \text { (AST) }\end{array}$ & $\begin{array}{l}\text { (Velho et al, } \\
\text { 2017). }\end{array}$ & 0-35 U/L & $15,1 \pm 3,9$ & $21,4 \pm 22,7$ \\
\hline $\begin{array}{l}\text { Gama glutamil } \\
\text { transpeptidase }\end{array}$ & $\begin{array}{l}\text { (Velho et al, } \\
\text { 2017). }\end{array}$ & 8-78 U/L & $11,4 \pm 2,3$ & $17,8 \pm 12,4$ \\
\hline Colesterol Total & $\begin{array}{l}\text { (Mueller et al, } \\
2007 \text { ) } \\
\text { (Jacobeit et al, } \\
2009 \text { ) } \\
\text { (Quirós et al, } \\
2015 \text { ) } \\
\text { Média }\end{array}$ & $<190 \mathrm{mg} / \mathrm{dL}$ & & $\begin{array}{l}189,13 \\
\mathrm{mg} / \mathrm{dL} \\
203 \mathrm{mg} / \mathrm{dL} \\
170,8 \mathrm{mg} / \mathrm{dL} \\
187,64 \\
\mathrm{mg} / \mathrm{dL}\end{array}$ \\
\hline HDL & $\begin{array}{l}\text { (Mueller et al, } \\
2007 \text { ) } \\
\text { (Jacobeit et al, } \\
2009 \text { ) } \\
\text { (Quirós et al, } \\
2015 \text { ) } \\
\text { Média }\end{array}$ & $>40 \mathrm{mg} / \mathrm{dL}$ & & $\begin{array}{l}53,64 \mathrm{mg} / \mathrm{dL} \\
50,5 \mathrm{mg} / \mathrm{dL} \\
48,8 \mathrm{mg} / \mathrm{dL} \\
50,98 \\
\mathrm{mg} / \mathrm{dL}\end{array}$ \\
\hline LDL & $\begin{array}{l}\text { (Mueller et al, } \\
2007 \text { ) } \\
\text { (Jacobeit et al, } \\
2009 \text { ) } \\
\text { (Quirós et al, } \\
2015 \text { ) } \\
\text { Média }\end{array}$ & $\begin{array}{l}<130 \\
\mathrm{mg} / \mathrm{dL}\end{array}$ & & $\begin{array}{l}130,04 \\
\mathrm{mg} / \mathrm{dL} \\
129 \mathrm{mg} / \mathrm{dL} \\
108,3 \mathrm{mg} / \mathrm{dL} \\
\mathbf{1 2 2 , 4 4} \\
\mathbf{m g} / \mathbf{d L}\end{array}$ \\
\hline Triglicerídeos & $\begin{array}{l}\text { (Mueller et al, } \\
\text { 2007) }\end{array}$ & $\begin{array}{l}<\quad 175 \\
\text { (Jejum) }\end{array}$ & & $\begin{array}{l}137.28 \\
\mathrm{mg} / \mathrm{dL}\end{array}$ \\
\hline
\end{tabular}


(Jacobeit et al, 2009)

(Quirós et al,

2015)
$87,5 \mathrm{mg} / \mathrm{dL}$ $86,3 \mathrm{mg} / \mathrm{dL}$ 103,69 $\mathrm{mg} / \mathrm{dL}$

Média

Fonte: elaborada pelo próprio autor.

\section{Perfil Glicêmico}

O perfil glicêmico apresenta uma diminuição dos níveis da glicose em jejum em homens trans em $\mathrm{TH}$, esta diminuição vem acompanhada pela relatada diminuição da sensibilidade à insulina, gerando um aumento compensativo deste hormônio no plasma durante o jejum (GOOREN; GILTAY; BUNCK, 2008). Os valores médios encontrados relacionados à glicemia em jejum neste grupo foram de 81 a 93,6 mg/dL, sendo o valor de referência para este parâmetro $<100 \mathrm{mg} / \mathrm{dL}$ ("Diretrizes da SBD 2019-2020", 2020).

\section{Perfil Hormonal}

As alterações encontradas no perfil hormonal são as mais relevantes deste grupo e estão diretamente ligadas à administração de testosterona exógena. Os níveis de hormônios sexuais no soro podem sofrer alterações conforme a via de administração do hormônio. As dosagens do hormônio podem ser adequadas conforme os testes laboratoriais do paciente, o excesso de testosterona acarreta na conversão da mesma em estrogênio por aromatização, gerando um efeito contrário ao desejado (UNGER, 2016). Os valores médios de hormônios sexuais (testosterona e estradiol) encontrados na literatura podem ser visualizados na Tabela 3.

Tabela 3: Média dos valores de hormônios sexuais no soro de homens trans pré e pós terapia hormonal, segundo a literatura ${ }^{18}$.

\begin{tabular}{|l|l|l|l|l|}
\hline Perfil & Valores & de & Valores Pré & Valores Pós TH \\
Sistêmico & Referência & TH & \\
\hline
\end{tabular}




\begin{tabular}{|c|c|c|c|c|}
\hline Testosterona & $\begin{array}{l}\text { (Vita et al, } \\
2018 \text { ) }\end{array}$ & 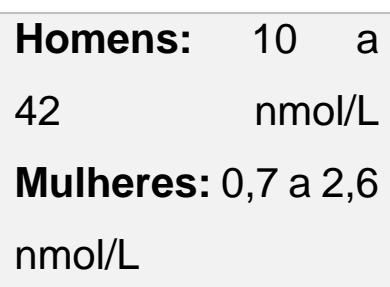 & $\begin{array}{l}1.6 \pm 0.5 \\
\mathrm{nmol} / \mathrm{L}\end{array}$ & $17.0 \pm 8.5 \mathrm{nmol} / \mathrm{L}$ \\
\hline Estradiol & $\begin{array}{l}\text { (Vita et al, } \\
2018 \text { ) }\end{array}$ & $\begin{array}{l}\text { Mulheres: } 50-150 \\
\text { pmol/L } \\
\text { Homens: } \\
110 \mathrm{pmol} / \mathrm{L}\end{array}$ & $\begin{array}{l}164.5 \pm 54.3 \\
\mathrm{pmol} / \mathrm{L}\end{array}$ & $\begin{array}{l}220.6 \pm 98.7 \\
\mathrm{pmol} / \mathrm{L}\end{array}$ \\
\hline
\end{tabular}

Fonte: elaborada pelo próprio autor.

Levando em consideração estes valores de referência e os dados coletados quanto ao perfil hormonal deste grupo, é seguro dizer que ocorreu a inversão desejada dos valores da testosterona, embora com isso também tenha havido o aumento do estradiol como mecanismo compensatório do corpo, por conta dos picos esporádicos em época de administração do hormônio (UNGER, 2016; GOOREN; GILTAY; BUNCK, 2008).

\subsection{EFEITOS ADVERSOS DA TERAPIA HORMONAL}

A terapia hormonal com testosterona é considerada segura, se os níveis deste hormônio se mantiverem a nível fisiológico, dentro dos valores de referência masculinos. Estudos sugerem, porém, que um aumento brusco da testosterona a nível suprafisiológico pode afetar negativamente os índices que podem levar a patologias cardíacas (GOOREN, 2014). A administração de testosterona a longo termo, segundo a literatura, pode acarretar na diminuição do $\mathrm{HDL}$ e no aumento de LDL e triglicerídeos, assim como no aumento de marcadores de inflamação e da pressão arterial (UNGER, 2016; GOOREN, 2014)

\section{CONSIDERAÇÕES FINAIS}

Com relação aos resultados descritos pela literatura científica, é possível sinalizar que em função da terapia hormonal com testosterona os valores em todos os parâmetros 
sistêmicos mostram algum nível de alteração causada pela administração do hormônio.

As alterações sistêmicas desencadeiam as mudanças morfofisiológicas e comportamentais decorrentes da reposição hormonal. De forma geral, os valores encontrados mostram que em todos os parâmetros que utilizam do sistema binário de divisão de valores de referência, ocorreu a inversão dos valores, indo do feminino ao masculino, com a administração continua da terapia hormonal.

Esse estudo, portanto, trouxe evidências das mudanças dos parâmetros sistêmicos, contribuindo de maneira significativa aos usuários da $\mathrm{TH}$, relatando as profundas mudanças pelas quais seus corpos irão passar. O conhecimento destas alterações se faz muito necessária, para que fiquem claras as alterações corporais e sistêmicas que ocorrem, servindo apoio no momento da decisão de dar início ou não ao processo de transição. Os resultados do estudo também podem ser utilizados futuramente como ferramenta de amparo científico para profissionais da saúde que se depararem com exames de pacientes trans masculinos, dando assim maior segurança e dignidade em diagnósticos de homens em TH.

\section{REFERÊNCIAS}

ADRIAANSEN, M. et al. Binary male-female laboratory reference ranges do not reflect reality for transgender individuals on sex-hormone therapy. New Zealand Journal of Medical Laboratory Science, v. 71, n. 3, p. 101-05, 2017.

BAO, A.; SWAAB, D. Sexual differentiation of the human brain: Relation to gender identity, sexual orientation and neuropsychiatric disorders. Frontiers in Neuroendocrinology, v. 32, n. 2, p. 214-26, 2011.

BENTZ, E. et al. A polymorphism of the CYP17 gene related to sex steroid metabolism is associated with female-to-male but not male-to-female transsexualism. Fertility and Sterility, v. 90, n. 1, p. 56-9, 2008. 
BURKE, S. et al. Testosterone Effects on the Brain in Transgender Men. Cerebral Cortex, v. 28, n. 5, p. 1582-96, 2017.

CHIPKIN, S.; KIM, F. Ten Most Important Things to Know About Caring for Transgender Patients. The American Journal of Medicine, v. 130, n. 11, p. 1238-1245, 2017.

CID10 Codigo Internacional de Doencas. Disponível em: <https://www.cid10.com.br/buscacode?query=F640>. Acesso em: 10 nov. 2020.

Diretrizes da 2019-2020. DBD Disponível em: $<$ https://www.diabetes.org.br/profissionais/images/DIRETRIZES-COMPLETA-20192020.pdf>. Acesso em: 10 nov. 2020.

FERNANDEZ, J.; TANNOCK, L. Metabolic effects of hormone therapy in transgender patients. Endocrine Practice, v. 22, n. 4, p. 383-388, 2016.

GANDHI, P.; MEDEIROS, E.; SHAH, A. Physiology or Pathology? Elevated Serum Creatinine in a Female-to-Male Transgender Patient. American Journal of Kidney Diseases, v. 75, n. 4, p. A13-A14, 2020.

GOOREN, L. Management of female-to-male transgender persons. Current Opinion in Endocrinology \& Diabetes and Obesity, v. 21, n. 3, p. 233-38, 2014.

GOOREN, L.; GILTAY, E.; BUNCK, M. Long-Term Treatment of Transsexuals with Cross-Sex Hormones: Extensive Personal Experience. The Journal of Clinical Endocrinology \& Metabolism, v. 93, n. 1, p. 19-25, 2008.

GORTON, R.; ERICKSON-SCHROTH, L. Hormonal and Surgical Treatment Options for Transgender Men (Female-to-Male). Psychiatric Clinics of North America, v. 40, n. 1, p. 79-97, 2017.

GUPTA, S.; IMBOREK, K.; KRASOWSKI, M. Challenges in Transgender Healthcare: The Pathology Perspective. Laboratory Medicine, v. 47, n. 3, p. 180-88, 2016. 
IRWIG, M. Testosterone therapy for transgender men. The Lancet Diabetes \& Endocrinology, v. 5, n. 4, p. 301-311, 2017.

MSD Manuals. Disponível em: <https://www.msdmanuals.com>. Acesso em: 10 nov. 2020.

SARASWAT, A.; WEINAND, J.; SAFER, J. Evidence Supporting the Biologic Nature of Gender Identity. Endocrine Practice, v. 21, n. 2, p. 199-204, 2015.

SILVA, L. et al. Uso do nome social no Sistema Único de Saúde: elementos para o debate sobre a assistência prestada a travestis e transexuais. Physis: Revista de Saúde Coletiva, v. 27, n. 3, p. 835-846, 2017.

UNGER, C. Hormone therapy for transgender patients. Translational Andrology and Urology, v. 5, n. 6, p. 877-84, 2016.

URIBE, C. et al. Brain network interactions in transgender individuals with gender incongruence. Neurolmage, v. 211, p. 116613, 2020.

VELHO, I. et al. Effects of testosterone therapy on BMI, blood pressure, and laboratory profile of transgender men: a systematic review. Andrology, v. 5, n. 5, p. 881-88, 2017.

VITA, R. et al. Changes in hormonal and metabolic parameters in transgender subjects on cross-sex hormone therapy: A cohort study. Maturitas, v. 107, p. 92-6, 2018.

YOKOTA, Y.; KAWAMURA, Y.; KAMEYA, Y. Callosal Shapes at the Midsagittal Plane: MRI Differences of Normal Males, Normal Females, and GID. 2005 IEEE Engineering in Medicine and Biology 27th Annual Conference, 2005.

Enviado: Novembro, 2020

Aprovado: Novembro, 2020. 